\title{
Green Synthesis of silver nanoparticles involving extract of plants of different taxonomic groups
}

\begin{abstract}
Silver nanoparticles are being used in numerous technologies and incorporated into a wide array of consumer products that take advantage of their desirable optical, conductive, and antibacterial properties. Silver nanoparticles have attained a special focus due to its antimicrobial property. Conventionally silver nanoparticles are synthesized by chemical method using chemicals as reducing agents which later on become accountable for various biological risks due to their general toxicity; engendering the serious concern to develop environment friendly processes. Thus, to solve the objective; principles of green chemistry have now become a torch for chemical technologist, biotechnologist and nanotechnologist worldwide in developing less hazardous chemicals. The present review explores the synthesis of silver nanoparticles through a natural and single step protocol preparatory method using the different plant products of different texa belonging to different families with green principles over the conventional ones.
\end{abstract}

Volume 5 Issue 2 - 2017

\author{
Deepika Gupta,' Pratima Chauhan² \\ 'Environmental Sciences, Department of Botany, University Of \\ Allahabad, India \\ ${ }^{2}$ Department of Physics, University Of Allahabad, India
}

Correspondence: Pratima Chauhan, Department of Physics,
University Of Allahabad, Allahabad, UP, 2 I I 002, India Email mangul67@yahoo.co.in

Received: December 31, 2016 | Published: March 08, 2017

Keywords: Green synthesis, Silver nanoparticles, Plants extract, Precursor

\section{Introduction}

Nanotechnology today is regarded as a revolutionary technology which deals with the matter at nanoscale $(1-100 \mathrm{~nm})$. Within this size range all the properties (chemical, physical and biological) changes in fundamental ways of both individual atoms/molecules and their corresponding bulk. Novel applications of nanoparticles and nanomaterials are growing rapidly on various fronts due to their completely new or enhanced properties based on size, their distribution and morphology. Nanotechnology is emerging as the sixth revolutionary technology in the current era. It is now emerging and fast growing field of science which is being exploited over a wide range of disciplines such as physics, chemistry, biology, material science, electronics, medicine, energy, environment and health sectors. The nanoparticles used for all the aforesaid purposes, the metallic nanoparticles considered as the most promising as they contain remarkable antibacterial properties due to their large surface area to volume ratio. Amongst the all noble metal nanoparticles, silver nanoparticles are significant which has gained boundless interests because of their unique properties such as chemical stability, good conductivity, catalytic and most important antimicrobial and antiinflammatory activities ..$^{1,2}$ Silver's mode of action is presumed to be dependent on $\mathrm{Ag}+$ ions, which strongly inhibit bacterial growth through suppression of respiratory enzyme and electron transport components and through interference with DNA functions ${ }^{3}$

Because of their wide range of applications Synthesis of silver nanoparticles is of much interest to the researcher. Generally, nanoparticles are prepared by a variety of chemical and physical methods which are quite expensive and potentially hazardous to the environment which involve use of toxic chemicals that are responsible for various biological risks. In the search of cheaper and ecocompatible pathways for nanoparticles, scientist used microorganism ${ }^{4-11}$ and plant extracts.$^{12-15}$ Green synthesis of nanoparticles has proven to be better methods due to slower kinetics, offer better manipulation, control over crystal growth and their stabilization. Greener synthesis provides advancement over traditionally used nanoparticles synthesis methods i.e. chemical. ${ }^{16,17}$ and physical method as it is cost effective, easily scaled up, environment friendly.$^{18}$ or large scale synthesis and in this method there is no need to use toxic chemicals. Green synthesis of nanoparticles is a bottom up approach where the main reaction occurring is reduction. Biogenic synthesis is useful not only because of its reduced environmental impact. ${ }^{19-21}$ compared with some of the physicochemical production methods, but also because it can be used to produce large quantities of nanoparticles that are free of contamination and have a well-defined size and morphology .$^{22}$ Biosynthetic routes can actually provide nanoparticles of a better defined size and morphology than some of the physicochemical methods of production..$^{23}$ The methods for obtaining nanoparticles using naturally occurring reagents such as vitamins, sugars, plant extracts, biodegradable polymers, and microorganism as reductants and capping agents could be considered attractive for nanotechnology. But among above mentioned reagents plant extract using leaf, root, stem, latex, resin, seed seems to be the best candidates and they are suitable for large scale "Green synthesis" of nanoparticles. The advancement of green syntheses over chemical and physical methods is: environment friendly, cost effective and easily scaled up for large scale syntheses of nanoparticles, furthermore there is no need to use high temperature, pressure, energy and toxic chemicals. ${ }^{24}$ Although, among the various biological methods of silver nanoparticle synthesis, microbe mediated synthesis is not of industrial feasibility due to the requirements of highly aseptic conditions and their maintenance. Therefore; the use of plant extracts for this purpose is potentially advantageous over microorganisms due to the ease of improvement, the less biohazard and elaborate process of maintaining cell cultures 25

Hence, a review is compiled describing the green syntheses of silver nanoparticles that provide advancement over conventional methods as it is cost effective and lesser or almost zero contaminations for the environment.

\section{Green synthesis of silver nps by plants}

A large number of plants are reported to facilitate silver nanoparticles syntheses are mentioned in Table 1 and are discussed briefly in the presented review. 
Table I Use of different plant parts extract in the synthesis of silver nanoparticles as a precursor

\begin{tabular}{|c|c|c|c|c|}
\hline $\begin{array}{l}\text { S. No. } \\
\text { Leaves }\end{array}$ & Latin Name & Family Name & Size and Shape & References \\
\hline $\mathrm{I}$ & Azadirachta indica & Meliaceae & spherical & 21 \\
\hline 2 & Aloe vera & Xanthorrhoeaceae & $12.2 \mathrm{~nm}$, spherical & 42 \\
\hline 3 & Argemone maxicana & Papaveraceae & I6-40nm, crystalline & 68 \\
\hline 4 & Tea & Theaceae & 20 to $90 \mathrm{~nm}$ & 69 \\
\hline 5 & Citrullus colocynthis & Cucurbitaceae & $3 \mathrm{Inm}$ & 49 \\
\hline 6 & Cassia auriculata & Caesalpiniaceae & $20-40 \mathrm{~nm}$ & 70 \\
\hline 7 & Coleus aromaticus & Labiatae & $44 \mathrm{~nm}$ & 51 \\
\hline 8 & Myrica nagi & Myricaceae & $50-69 \mathrm{~nm}$ & 71 \\
\hline 9 & Diopyros kaki & Ebenaceae & I5-500nm, cubic & 45 \\
\hline 10 & Euphorbia hirta & Euphorbiaceae & 40-50nm, spherical & 72 \\
\hline II & Ginko biloba & Ginkgoaceae & I5-500nm, cubic & 45 \\
\hline 12 & Helianthus annus & Asteraceae & & 44 \\
\hline 13 & Hibiscus rosasinensis & Malvaceae & I3nm, spherical & 73 \\
\hline 14 & Magnolia kobus & Magnoliaceae & I5-500nm, cubic & 45 \\
\hline 15 & Mangifera indica & Anacardiaceae & $\begin{array}{l}\text { 20nm, spherical, triangular, } \\
\text { hexagonal }\end{array}$ & 74 \\
\hline 16 & Mentha piperita & Lamiaceae & $90 \mathrm{~nm}$, spherical & 76 \\
\hline 17 & Memecylon edule & Melastomataceae & $50-90 \mathrm{~nm}$, square & 77 \\
\hline 18 & Murraya keenigii & Rutaceae & IOnm,crystalline, spherical & 75 \\
\hline 19 & Nicotiana tobaccum & Solanaceae & 8nm, crystalline & 78 \\
\hline 20 & Ocimum tenuiflorum & Lamiaceae & 5-10nm, spherical & 26 \\
\hline 21 & Oryza sativa & Poaceae & & 44 \\
\hline 22 & Pelargonium graveolens & Geraniaceae & I6-40nm, crystalline & 41 \\
\hline 23 & Piper betle & Piperaceae & 3-37nm, spherical & 79 \\
\hline 24 & Platanus orientalis & Platanaceae & I5-500nm, cubic & 47 \\
\hline 25 & Pinus desiflora & Pinaceae & I5-500nm, cubic & 47 \\
\hline 26 & Rosa rugosa & Rosaceae & $30-60 \mathrm{~nm}$ & 80 \\
\hline 27 & Saccharum officinarum & Poaceae & & 44 \\
\hline 28 & Sorghum bicolour & Poaceae & & 44 \\
\hline \multicolumn{5}{|c|}{ Seed Extract } \\
\hline I & Jatropha curcas & Euphorbiaceae & I0-20nm, crystalline & 63 \\
\hline 2 & Medicago sativa & Fabaceae & 5-5 Inm, spherical & 65 \\
\hline 3 & Papaver somniferum & Papaveraceae & & 64 \\
\hline 4 & Nyctanthes arbor-tristis & Nyctanthes & 50 and $80 \mathrm{~nm}$, spherical & 15 \\
\hline \multicolumn{5}{|c|}{ Fruit Extract } \\
\hline I & Emblica officinalis & Euphorbiaceae & $10-20 \mathrm{~nm}$ & 38 \\
\hline 2 & Carica papaya & Caricaceae & I5nm, cubic & 12 \\
\hline 3 & Tanacetum vulgare & Asteraceae & I6nm, spherical & 80 \\
\hline \multicolumn{5}{|c|}{ Fruit Peel Extract } \\
\hline I & Musa pudica & Musaceae & $20 \mathrm{~nm}$ & 35 \\
\hline 2 & Annona squamosa & Annonaceae & 20-60nm, spherical & 36 \\
\hline 3 & Citrus sinensis & Rutaceae & I4-20nm, spherical & 81 \\
\hline 4 & Punica granatum & Punicaceae & $5+1.5 \mathrm{~nm}$ & 37 \\
\hline \multicolumn{5}{|c|}{ Gum and Latex } \\
\hline I & Jatropha curcas & Euphorbiaceae & & 79 \\
\hline 2 & Euphorbia & Euphorbiaceae & $18 \mathrm{~nm}$ & 26 \\
\hline 3 & Acacia & Mimosoideae & & 28 \\
\hline 4 & Boswellia serrata & Burseraceae & & 27 \\
\hline 5 & Peach gum & Rosaceae & $23.56 \pm 7.87 \mathrm{~nm}$ & 80 \\
\hline \multicolumn{5}{|c|}{ Bark/ Stem Powder } \\
\hline I & Cinnamon zeylanicum & Lauraceae & 3I-40nm, spherical & 60 \\
\hline 2 & Shorea tumbuggaia & Dipterocarpaceae & Spherical & 61 \\
\hline 3 & Boswellia ovalifoliolata & Burseraceae & Spherical & 61 \\
\hline \multicolumn{5}{|c|}{ Tuber, Root, Rhizome } \\
\hline 1 & Curcuma longa & Zingiberaceae & $\begin{array}{l}21-30 \mathrm{~nm} \text {, quasi-spherical, } \\
\text { triangular }\end{array}$ & 58 \\
\hline 2 & Dioscorea bulbifera & Dioscoreaceae & $8-20 \mathrm{~nm}$, spherical, triangular & 56 \\
\hline 3 & Ocimum sanctum & Lamiaceae & 5-10nm, spherical & 82 \\
\hline 4 & Zingiber offcinale & Zingiberaceae & 6-20nm, spherical & 82 \\
\hline \multicolumn{5}{|c|}{ Coir Extract } \\
\hline I & Cocos nucifera & Arecaceae & $23+2 n m$ & 55 \\
\hline \multicolumn{5}{|c|}{ Flower Extract } \\
\hline I & Pandanus odorifer Forssk (spath of male inflorescence) & Pandanaceae & 24-55 nm, quasispherical & 66 \\
\hline 2 & Hibiscus sabdariffa (flower) & Malvaceae & & 67 \\
\hline
\end{tabular}




\section{Preparation of plant broth and biosynthesis of silver nanoparticles}

The protocol for the nanoparticle syntheses involves: the collection of the part of plant of interest from the available sites then it's washing thoroughly with tap water to remove contamination followed by surface sterilization with double distilled water and air dried at room temperature. These clean and fresh sources are then powdered using domestic blender or cut it into very small pieces. And for the plant broth preparation, around $10-25 \mathrm{~g}$ of the dried powder or finally chopped leaves were kept in a beaker and boiled with $100 \mathrm{~mL}$ of deionised distilled water. The extract was filtered with Whatman filter paper No.1 further the filtrate was used as reducing source for the synthesis of silver nanoparticles.

Synthesis of silver nanoparticles was carried out by adding $10 \mathrm{ml}$ of leaf extract to $100 \mathrm{ml}$ of $1 \mathrm{mM}$ silver nitrate $\left(\mathrm{AgNO}_{3}\right)$ solution with continuous stirring at room temperature. Reduction of $\mathrm{Ag}^{+}$to $\mathrm{AgO}$ was confirmed by the colour change of solution from colourless to brown. Its formation was further confirmed by using UV-Visible spectroscopy.

\section{Plant latex and gum as medium}

Synthesized the silver nanoparticles in one-step solvent free condition using Euphorbiaceae plant latex. ${ }^{26}$ Around eight plant species were utilized for the synthesis of nanoparticles, out of which Jatropha gossypifolia, Jatropha curcas, and Euphorbia milii showed an average of $62+105 \mathrm{~nm}$. The stem latex of Boswellia serrrata was successfully used to induce synthesis of silver nanoparticles. ${ }^{27}$ It has been demonstrated that the plant based exudates gum such as gum Acacia can be utilized as a reducing and stabilizing agent for the silver nanoparticle biosynthesis.$^{28}$ Gum kondagogu biopolymer derived as exudates from the bark of Cochlospermum gossypium used as a template for the synthesis and stabilization of silver nanoparticles. The synthesis was carried out in aqueous medium without the requirement of any added chemical reducing agent by autoclaving. Gum olibanum is a naturally occurring gum-oleo-resin derived as exudates from the bark of Boswellia serrata, a native tree of India. Besides its use as incense, fumigant and multipurpose aromatic; it is also exploited in silver nanoparticle synthesis as reducing and capping agent. Typically the gum consists of volatile oil, water soluble gum (polysaccharides), lipophilic terpenes and insoluble matter. The polysaccharide is abundant in neutral sugars and composed of galactose, arabinose, xylose and d-glucuronic acid. ${ }^{29,30}$ From the Raman spectrum of the nanoparticle solution it is confirmed that both amino and carboxylate groups of the gum are involved in the capping of the nanoparticles.$^{27}$

\section{Fruit peel extracts as medium}

Literature survey has shown that naturally available agricultural wastes have not been investigated for the synthesis of silver nanoparticles. A classical example of such an abundantly available natural material is the banana peel. Bananas are consumed all over the world. After consumption of the pulp, the peels are generally discarded. Beside a few applications of banana peels i.e. exploitation of their medicinal properties. ${ }^{31}$ in ethanol fermentation. ${ }^{32}$ as a substrate for generating fungal biomass. ${ }^{33}$ utilization as a biosorbent for heavy metal removal.$^{34,35}$ utilizes banana peel for the synthesis of silver nanoparticles.$^{36}$ employing peel extract of Annona squamosa for the synthesis of silver nanoparticles. Controlled growth of silver nanoparticles was formed in $4 \mathrm{hr}$ at room temperature $\left(25^{\circ} \mathrm{C}\right)$ and $60^{\circ} \mathrm{C}$. silver nanoparticles were irregular spherical in shape and the average particle size was about $35 \pm 5 \mathrm{~nm}$. The water soluble ketone and hydroxyl as functional group containing compounds are reported to be responsible for the reduction of silver ions. ${ }^{36}$ The Pomegranate fruit extract is a rich source of highly potent antioxidants due to rich in phenolic compounds (mainly Ellagic acid) employed for the synthesis of silver nanoparticles. Ellagic acid an active constituent present in fruit peel has an easy electron loosing capacity which results in the formation of $\mathrm{H}+$ radical, which reduces the size of silver to nano size. The morphological and crystalline phase study of the NPs showed that the average size of silver nanoparticles obtained from was $5 \pm$ $1.5 \mathrm{~nm} \cdot{ }^{37}$

\section{Fruit extract as medium}

On treating aqueous silver sulfate and chloroauric acid solutions with Emblica Officinalis fruit extract, rapid reduction of the silver and chloroaurate ions is observed leading to the formation of highly stable silver and gold nanoparticles in solution. Transmission Electron Microscopy analysis of the silver and gold nanoparticles indicated that they ranged in size from 10 to $20 \mathrm{~nm}$ and 15 to $25 \mathrm{~nm}$ respectively. ${ }^{38}$ The fruit extract of papaya works as reducing as well as capping agent. Nanoparticles on characterization analysis showed the average particle size of $15 \mathrm{~nm}$ as well as revealed their cubic structure. C-O group of polyols such as hydroxyl flavones and catechins present in green unripe Papaya (Carica papaya) fruit are mainly responsible for the reduction of Ag ions, whereby they themselves get oxidized to unsaturated carbonyl groups.$^{12}$

\section{Leaf extract as medium}

The synthesis of quasi-spherical silver nanoparticles and triangular or spherical gold nanoparticles using sun dried Cinnamomum camphora leaf without addition of any protectors or accelerators.$^{39}$ They demonstrated that the polyol components and water soluble hydrocyclic compounds present in leaf were mainly responsible for reduction of silver ions or chloroaurate ions. ${ }^{40}$ Reported the possibility of terpenoids from geranium leaf in the synthesis of nano-sized silver particles. ${ }^{41}$ Reported the synthesis of highly stable and crystalline silver nanoparticles $(16-40 \mathrm{~nm})$ by exposing the aqueous geranium leaf extract to silver nitrate solution. Highly concentrated silver nanoparticles obtained from the aqueous leaf extract of Azadirachta indica.$^{21}$ Leela A \& Song JY ${ }^{42,43}$ reported the synthesis of silver nanoparticles from the leaf extracts of Aloe vera and Capsicum annum plants, respectively. Among the leaf extracts of plants, namely, Helianthus annus, Basella alba, Oryza sativa, Saccharum officinarum, Sorghum bicolar, and Zea mays, it is concluded that among all the tested plant extracts, $H$. annus exhibited the strongest potential for rapid reduction of silver ions. ${ }^{44}$ Leaf extract of Pine, Persimmon, Ginkgo, Magnolia, and Platanus plants is used for the extracellular synthesis of silver nanoparticles. ${ }^{45}$ Methanolic extract of Eucalyptus hybrid leaves is exploited in the extracellular biosynthesis of silver nanoparticles. ${ }^{46}$ Similarly, Satyavani K .${ }^{47}$ reported rapid synthesis (reaction time $<30 \mathrm{~min}$ ) of silver nanoparticles using Acalypha indica leaf extract and their antibacterial activity against water borne pathogens. Patil RS. ${ }^{48}$ Highlighted the possibility of tissue culture-derived callus extract from Sesuvium portulacastrum for the synthesis of antimicrobial silver nanoparticles. Similarly, ${ }^{49}$ reported the synthesis of silver nanoparticles using the stem-derived callus extract of the bitter apple plant and illustrated their tremendous antibacterial activity. Very recently, bio-inspired synthesis of highly stabilized silver nanoparticles using Ocimum tenuiflorum as well as Coleus aromaticus have been reported by. ${ }^{31,50,51}$ respectively. Kulkarni AP. ${ }^{52}$ Synthesized silver nanoparticles with an average size of $35 \mathrm{~nm}$ using aqueous leaf extract of Catharanthus roseus and proven their activity against malaria parasite. SM Roopana ${ }^{53}$ reported the synthesis of silver nanoparticles using Piper longum leaf extracts. 
The particles had a uniform spherical shape and ranged in size from about 18 to $41 \mathrm{~nm}$. These nanoparticles were found to have a significant cytotoxic effect on HEp-2 cancer cells. In addition to the vast number of reports on angiospermic plants bryophytes are also utilized to synthesise silver nanoparticles. Recently Sougata Ghosh. ${ }^{54}$ reported synthesis of silver nanoparticles using the alcoholic extract of a Riccia a bryophyte.

\section{Coconut coir as medium}

Gole A..$^{55}$ reported that the reduction of silver ions occurred when silver nitrate solution was treated with aqueous extract of Cocus nucifera coir at $60^{\circ} \mathrm{C}$, particle synthesised with range of the size as $23 \pm 2 \mathrm{~nm}$ and face centred cubic silver nanoparticles obtained.

\section{Tuber, rhizome and root extract as medium}

Dioscorea bulbifera tuber extract is rich in flavonoid, phenolics, reducing sugars, starch, diosgenin, ascorbic acid, and citric acid. ${ }^{56}$ Energy dispersive $\mathrm{x}$-ray spectroscopy results confirmed the presence of significant amounts of silver with no contaminants and HRTEM images clearly show that the shape of silver nanoparticles were mostly spherical with dimensions of $75 \mathrm{~nm}$. In Curcuma longa terpenoids are believed to play an important role in silver nanoparticle biosynthesis through the reduction of silver ions. ${ }^{40,57,58}$ Ag-NPs with an average size of $6.30 \pm 2.64 \mathrm{~nm}$ and spherical shapes were synthesized using aqueous tuber-powder extract of C. Longa. ${ }^{59}$

\section{Bark extract as medium}

The compatibility of the bark and powder extracts of Cinnamon zeylanicum toward the formation of silver nanoparticles results that bark extract could produce a higher amount of silver nanoparticles compared to the powder extract. The resulting nanoparticles varied in shape and size but had strong antibacterial activity against the Escherichia coli. ${ }^{60}$ Savithramma N. ${ }^{59}$ Used bark extracts of Boswellia ovalifoliolata and Shorea tumbuggaia to synthesise silver nanoparticle.

\section{Seed extract as medium}

Joyita Banerjee. ${ }^{60}$ reported the formation of crystalline silver nanoparticles using seed extract of Syzygium cumuni. Harekrishna Bar ${ }^{61}$ reported that aqueous seed extract of Jatropha curcas can be used for both reducing silver ion to silver and stabilizing the particles during the growth process. They shows that size of the particles can be controlled within certain range from $15-50 \mathrm{~nm}$ by varying the concentration of $\mathrm{AgNO}_{3}$. Vijayaraghavan $\mathrm{K}^{62}$ reported synthesis of silver nanoparticles by using seed extract of Trachyspermum ammi (ajwain) and Papaver somniferum. The extracts of both T. ammi and P. somniferum showed a maximum absorbance at $430 \mathrm{~nm}$, as the reductant concentration increased the colour intensity also increased ${ }^{60}$ Audra I Lukman.${ }^{63}$ reported that colloidal silver $(\mathrm{Ag})$ nanoparticles were synthesized by reacting aqueous $\mathrm{AgNO}_{3}$ with Medicago sativa seed exudates under non-photomediated conditions. Upon contact, rapid reduction of $\mathrm{Ag}^{+}$ions was observed in $<1$ min with $\mathrm{Ag}$ nanoparticle formation reaching $90 \%$ completion in $<50 \mathrm{~min}$. Effect of $\mathrm{Ag}$ ion concentration, quantity of exudates and $\mathrm{pH}$ on the particle size and shape were investigated. At $\left[\mathrm{Ag}^{+}\right]=0.01 \mathrm{M}$ and $30^{\circ} \mathrm{C}$, largely spherical nanoparticles with diameters in the range of 5-51 nm were generated, while flower-like particle clusters $($ mean size $=104 \mathrm{~nm})$ were observed on treatment at higher Ag concentrations.

\section{Flower extract as medium}

The synthesis of silver nanoparticles using a broth prepared from the aromatic spath of male inflorescence of screw pine Pandanus odorifer (Forssk).$^{64}$ Flower extract of Hibiscus sabdariffa extracellular synthesized silver nanoparticle of $25 \mathrm{~nm} .{ }^{65}$

\section{Conclusion}

As metal nanoparticles seems to fascinate for the future diverse industry due to their enriched chemical, electrical and physical properties. The development of immaculate protocols for the synthesis of highly monodisperse nanoparticles of various sizes, geometries and chemical composition is one of the most challenging obstructions in the field of nanotechnology. The use of toxic chemicals and nonpolar solvents in synthesis leads to the inability to use nanoparticles in clinical fields. Therefore, development of clean, non-toxic, biocompatible and eco-friendly method for synthesis of nanoparticles deserves recognition. So there is need of eco friendly nanoparticles synthesis approach.

Massive numbers of plant species are available in nature, and many of them have huge potential for the production of nanomaterials. For the syntheses of nanoparticles employing plants can be advantageous over other biological entities which can overcome the time consuming process of employing microbes and maintaining their culture which can lose their potential towards synthesis of nanoparticles. And the advantages of using plants for the synthesis of nanoparticles are that the plants are easily available and safe to handle and possess a large variety of active agents that can promote the reduction of silver ions. Most of the plant parts like leaves, roots, latex, bark, stem, and seeds are being used for nanoparticle synthesis. Green synthesis silver nanoparticles using plant extracts provides benefits over chemical and physical method as it is economical, energy efficient, cost effective; provide healthier work places and communities, protecting human health and environment leading to lesser waste and safer products. This eco-friendly method can potentially be used in various areas, including pharmaceuticals, cosmetics, foods, and medical applications. Hence in this regard; use of plant extract for synthesis can form an immense impact in coming decades.

\section{Acknowledgements}

Support from the University of Allahabad, University Grant Commission (UGC) is gratefully acknowledged.

\section{Conflicts of interest}

None.

\section{References}

1. Simsikova M Interaction of graphene oxide with albumins: Effect of size, pH and temperature. Arch Biochem Biophys. 2016;593:69-79.

2. Lee DY, Khatun Z, Lee JH et al. Blood compatible graphene/heparin conjugate through noncovalent chemistry. Biomacromolecules. 2011;12(2):336-341.

3. Hassan M, Walter M, Moseler M Interactions of polymers with reduced graphene oxide: van der Waals binding energies of benzene on graphene with defects. Phys Chem Phys. 2014;16(1):33-37.

4. Baweja L, Balamurugan K, Subramanian V et al. Effect of graphene oxide on the conformational transitions of amyloid beta peptide: A molecular dynamics simulation study. J Mol Graph Model. 2015;61:175-185.

5. Alwarappan S, Boyapalle S, Kumar A et al. Comparative study of single-, few-, and multilayered graphene toward enzyme conjugation and electrochemical response. J Phys Chem C. 2012;116(11):6556-6559.

6. De M, Chou SS, Dravid VP Graphene oxide as an enzyme inhibitor: modulation of activity of $\alpha$-chymotrypsin. $J$ Am Chem Soc. 2011;133(44):17524-17527. 
7. Zhang Y, Zhang J, Huang X et al. Assembly of graphene oxide-enzyme conjugates through hydrophobic interaction. Small. 2012;8(1):154-159.

8. Shen J, Shi M, Yan B et al. Covalent attaching protein to graphene oxide via diimide-activated amidation. Colloids Surf B Biointerfaces. 2010;81(2):434-438.

9. Kou L, He H, Gao C Click chemistry approach to functionalize twodimensional macromolecules of graphene oxide nanosheets. NanoMicro Letters. 2010;2(3):177-183.

10. Guo C, Book-Newell B, Irudayaraj J Protein-directed reduction of graphene oxide and intracellular imaging. Chem Commun (Camb). 2011;47(47):12658-12660.

11. Li C, Adamcik J, Mezzenga R Biodegradable nanocomposites of Amyloid fibrils and graphene with shape-memory and enzyme-sensing properties. Nat Nanotechnol. 2012;7(7):421-427.

12. Xing R, Jiao T, Liu Y et al. Co-assembly of graphene oxide and albumin/ photosensitizer nanohybrids towards enhanced photodynamic therapy. Polymers. 2016;8(5):181.

13. Vardharajula S, Ali SZ, Tiwari PM et al. Functionalized carbon nanotubes: biomedical applications. Int J Nanomedicine. 2012;7(7):5361-5374.

14. Mahmoudi M, Akhavan O, Ghavami M et al. Graphene oxide strongly inhibits amyloid beta fibrillation. Nanoscale. 2012;4(23):7322-7325.
15. Li M, Yang X, Ren J et al. Using graphene oxide high near-infrared absorbance for photothermal treatment of Alzheimer's disease. Adv Mater. 2012;24(13):1722-1728.

16. Liu J, Fu S, Yuan B et al. Toward a universal "adhesive nanosheet" for the assembly of multiple nanoparticles based on a proteininduced reduction/decoration of graphene oxide. $J$ Am Chem Soc. 2010;132(21):7279-7281

17. Gully BS, Zou J, Cadby G et al. Colloidal graphenes as heterogeneous additives to enhance protein crystal yield. Nanoscale. 2012;4(17):5321-5324.

18. Govada L, Leese HS, Saridakis E et al. Exploring carbon nanomaterial diversity for nucleation of protein crystals. Sci Rep. 2016;6:20053.

19. Xue T, Jiang S, Qu Y et al. Graphene-supported hemin as a highly active biomimetic oxidation catalyst. Angew Chem Int Ed Engl. 2012;51(16):3822-3825.

20. Huang C, Bai H, Li C, Shi G A graphene oxide/haemoglobin composite hydrogel for enzymatic catalysis in organic solvents. Chem Commun (Camb). 2011;47(17):4962-4964. 